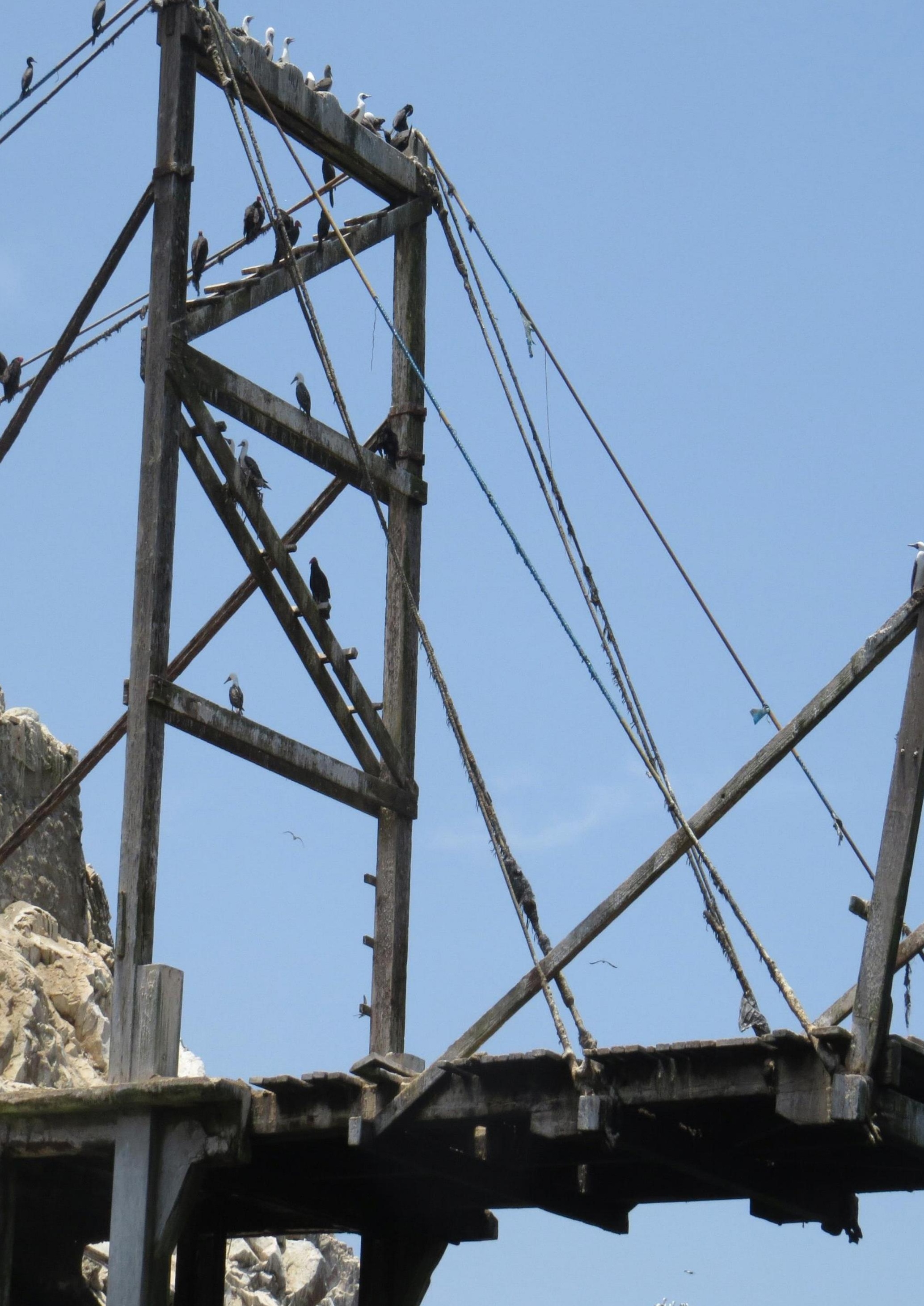




\title{
A IMPORTÂNCIA DA VISITAÇÃO NOS PARQUES NACIONAIS E A GERAÇÃO DE RECURSOS FINANCEIROS
}

\author{
The importance of the visitation to National Parks and the generation of \\ financial resources
}

Paula Oliveira Gomes ${ }^{1}$

André de Almeida Cunha ${ }^{2}$

\section{RESUMO}

O crescente interesse pelas áreas naturais tem gerado também maior preocupação com a preservação dos recursos ambientais. Nesse sentido, esse estudo apresenta a relação do homem com o meio ambiente e a relevância da geração de recursos financeiros a partir da visitação nos Parques Nacionais. Dessa maneira, a fim de verificar a importância financeira da manutenção das áreas protegidas, estudos vêm utilizando os métodos de valoração ambiental.

Palavras-chave: Unidades de Conservação. Sustentabilidade financeira. Valoração ambiental econômica.

\begin{abstract}
The growing interest in natural areas has also generated greater concern for the preservation of environmental resources. Therefore, this study presents the relation human with the environment and the importance of the generation of financial resources from the visitation in National Parks. Thus, in order to verify the financial importance of the maintenance of protected areas, studies have been applying the environmental valuation methods.
\end{abstract}

Keywords: Conservation Units. Financial sustainability. Environmental economic valuation.

\section{INTRODUÇÃO}

A conservação da biodiversidade de forma integrada com o desenvolvimento econômico e o bem-estar da população é uma maneira de assegurar a capacidade dos ecossistemas de suprir as necessidades atuais e futuras da sociedade e, assim, avançar rumo ao desenvolvimento sustentável (Guedes; Seehusen, 2011).

As discussões sobre estratégias para a conservação da biodiversidade in situ devem levar em conta também a questão da sustentabilidade financeira das áreas protegidas, o que pode gerar recursos financeiros adicionais para a conservação por meio da cobrança de taxa de ingresso, doações e venda de produtos locais (Medeiros et al., 2011).

\footnotetext{
${ }^{1}$ Mestra em Turismo. Centro de Excelência em Turismo da Universidade de Brasília. E-mail: paula.gomes.df@gmail.com

${ }^{2}$ Doutor em Ecologia, Conservação e Manejo da Vida Silvestre, UFMG. Professor Adjunto do Departamento de Ecologia da UnB, do Centro de Excelência em Turismo e do Centro de Estudos do Cerrado.E-mail: cunha.andre@gmail.com.
} 
Embora a maioria das Unidades de Conservação (UC) brasileiras não gerem receitas próprias, essas áreas são responsáveis por uma vasta gama de serviços ambientais, que são proporcionados ao ser humano por meio dos ecossistemas. Além disso, cumprem com os objetivos de conservação e desenvolvimento e contribuem, no curto e longo prazo, para o desenvolvimento econômico e social do Brasil (Medeiros et al., 2011).

No cenário mundial e especialmente brasileiro, as restrições orçamentárias comprometem objetivos básicos de conservação, como: a gestão das áreas protegidas, insuficiência orçamentária, recursos humanos e materiais precários, entraves administrativos, institucionais e políticos. Os orçamentos, insuficientes para atender as demandas e competências, comprometem a capacidade do setor ambiental em conceber, implantar e executar as políticas públicas de modo eficaz (Camphora, 2010).

Os dados orçamentários das UC expõem que a arrecadação obtida pelo poder público, por exemplo, por meio das concessões e cobrança de ingresso, pode incrementar os recursos para a manutenção e a gestão da visitação nestas áreas (Rodrigues; Godoy, 2013).

Ressalta-se que a importância das UC não é facilmente internalizada na economia, facilitando a substituição dessas áreas naturais pelo uso convencional do solo por atividades como o agronegócio. Desta forma, o turismo ao ser valorado pode demonstrar o quanto de benefícios econômicos, sociais e, em especial, a conservação da natureza, podem ser gerados a partir da visitação.

A razão desse estudo é justificada pela carência de dados sobre valoração econômica do turismo, relevantes para subsidiar as decisões dos governos sobre a gestão do uso público nos parques, objetivo esse que tem impulsionado pesquisas realizadas nos parques nacionais (Driml, 2010). Ademais são apresentados os conceitos de biologia da conservação e Unidades de Conservação, sustentabilidade financeira dos Parques Nacionais e métodos de valoração ambiental.

Salienta-se a importância dos benefícios econômicos oferecidos pelos serviços ecossistêmicos das áreas protegidas, a qual é observada a partir da valoração ambiental, especialmente com base nos métodos de Custo de Viagem e de Valoração Contingente.

\section{A BIOLOGIA DA CONSERVAÇÃO E A CRIAÇÃO DE UNIDADES DE CONSERVAÇÃO}

Diante do cenário de crise da biodiversidade e de forma a combatê-la ou ao menos mitigá-la, a biologia da conservação primeiramente busca entender os efeitos da atividade humana nas espécies, comunidades e ecossistemas, em seguida, desenvolve abordagens práticas para prevenir a extinção de espécies e, se possível, reintegrar as espécies ameaçadas ao seu ecossistema funcional (Primack; Rodrigues, 2001). 
A discussão sobre a biodiversidade é motivada pela preocupação com a destruição da natureza em ritmo acelerado, até mesmo pelo crescimento da população humana e das atividades econômicas, os quais ocasionam consumo intenso de recursos naturais e despejo de poluentes no ambiente (Ehrenfeld, 1997).

Basicamente, a degradação ambiental ocorre por razões econômicas, como o crescimento demográfico, a expansão das fronteiras agrícolas, a urbanização, a industrialização, e principalmente a obtenção de lucros. O aumento das ameaças à diversidade biológica é ocasionado também pela demanda de consumo da população humana, que cresce rapidamente, junto a contínuos avanços tecnológicos. A desigualdade na distribuição de renda no mundo torna a situação ainda pior (Primack; Rodrigues, 2001).

Uma das medidas encontradas para a preservação de ecossistemas e espécies é a criação das Unidades de Conservação (Primack; Rodrigues, 2001). A conservação das áreas naturais e o manejo de recursos sustentáveis são, portanto, essenciais para o planejamento, o desenvolvimento e o gerenciamento do ecoturismo (Wearing; Neil, 2014). Especialmente em ambientes onde há maior perda de biodiversidade, a criação e manutenção das UC são extremamente importantes (Millennium Ecosystem Assessment, 2005).

Na história recente, a origem das Áreas Protegidas ocorreu com a criação do Parque Nacional de Yellowstone em 1872, que marcou o interesse humano pela proteção de lugares especiais do planeta (Fonseca; Lamas; Kasecker, 2010). Na época, o fato causou controvérsia podendo ser, por um lado, uma ideia inspirada por altruísmo em favor de todas as gerações ou, por outro lado, influência de interesses comerciais por parte de empresas (Araújo, 2012).

No Brasil, as áreas protegidas adquiriram certo reconhecimento no decorrer do século XX. Antes disso, o País se focava principalmente no que era economicamente mais relevante, como o cultivo de madeira e a produção de minérios (Medeiros; Irving; Garay, 2004).

Inspirado na criação do Parque Nacional de Yellowstone, André Rebouças, pioneiro na ética conservacionista no Brasil, defendeu a necessidade de criar parques nacionais também no Brasil (Rylands; Brandon, 2005). Os cenários desfavoráveis impediram por vários anos que esse ideal se realizasse, e assim, somente no ano de 1937 foi criado o Parque Nacional de Itatiaia (Araújo, 2012).

Outro momento importante no país foi a promulgação da Constituição Federal de 1988, a qual previu a proteção ambiental a partir da criação de espaços protegidos (Brasil, 1988). O tema foi efetivado quando se criou a Lei no 9.985/2000, a qual estabeleceu o Sistema Nacional de Unidades de Conservação da Natureza (SNUC), apresentando um avanço importante na construção de um sistema efetivo de áreas protegidas brasileiras. E também se esclareceu o entendimento das UC, como espaço territorial e seus recursos ambientais, incluindo as águas jurisdicionais, com 
características naturais relevantes, legalmente instituído pelo Poder Público, com objetivos de conservação e limites definidos, sob regime especial de administração, ao qual se aplicam garantias adequadas de proteção (Brasil, 2000b).

A partir desse progresso do SNUC, proteger a natureza entrou definitivamente na agenda governamental brasileira, passando a configurar um objetivo complementar da política de desenvolvimento nacional (Medeiros; Irving; Garay, 2004), e a proteção e conservação da natureza adquiriu um novo status na política nacional, consistindo em tarefa ou dever a serem cumpridos e fiscalizados pelo Poder Público.

Outro marco para as UC administradas pela União, aconteceu no ano de 2008, em que essas áreas naturais passaram a ter orçamento próprio, desvinculado do orçamento dedicado a outros temas da gestão ambiental federal, o que possibilitou uma visão mais clara sobre o que efetivamente se investe anualmente nas UC federais (Brasil, 2009).

\section{OS PARQUES NACIONAIS E SUA SUSTENTABILIDADE FINANCEIRA}

Considerando o uso que lhes são permitidos, há dois grupos de UC: as Unidades de Uso Sustentável e as de Proteção Integral. Os Parques Nacionais são classificados como UC de Proteção Integral, e esse grupo têm como objetivo primordial preservar os recursos naturais, de modo a manter os ecossistemas protegidos da ação humana, controlando a exploração e o uso indireto dos recursos naturais (Brasil, 2000b), os quais relacionam-se às funções dos ecossistemas que beneficiam as pessoas indiretamente, como a purificação da água, formação do solo, polinização, controle biológico e regulação da composição dos gases atmosféricos (GUEDES; SEEHUSEN, 2011; MOTTA, 2006; TOLEDO, 1998).

Em especial os Parques Nacionais brasileiros tem enfoque na preservação de ecossistemas naturais de grande relevância ecológica e beleza cênica, possibilitando a realização de pesquisas científicas e o desenvolvimento de atividades de educação e interpretação ambiental, de recreação em contato com a natureza e de turismo ecológico (Brasil, 2000a).

Essas áreas podem contribuir efetivamente para o desenvolvimento do turismo, entretanto, fomentar o uso público e promover o turismo da região não são os únicos objetivos dessas áreas. Com destaque para os parques nacionais, o turista muitas vezes é atraído para apreciar a existência dos recursos naturais ali existentes (Pardini, 2012).

Ademais, o turismo como instrumento de conservação depende de um entendimento das expectativas dos visitantes, da adequação da área visitada e da capacidade dos gestores do parque para fornecer uma experiência de alta qualidade, ao mesmo tempo minimizando os potenciais impactos negativos da visitação (Eagles; Hillel, 2008). 
Uma vez que a visitação permite a sensibilização dos visitantes para a preservação e conservação das áreas naturais. Nessas áreas, criadas para conservar a biodiversidade e deter a perda em grande escala dos ecossistemas naturais, o turista tem a oportunidade de descobrir, participar e interagir ativamente com o meio ambiente, estimulando-o a assumir um papel proativo na criação de sua própria experiência (Wearing; Neil, 2014).

No entanto, a sociedade e o governo muitas vezes não têm conhecimento da relevância dos serviços ambientais existentes nas UC e o quanto podem gerar de contribuições econômicas que, quando monetizadas, superam significativamente o montante destinado pela administração pública à manutenção do SNUC (Medeiros et al., 2011).

Vale ressaltar que o financiamento oficial das UC a nível federal ocorre via quatro fontes principais: ICMBio, IBAMA, MMA e Serviço Florestal Brasileiro - SFB (Brasil, 2009). Nota-se, portanto, que a gestão pública das UC possui um grande entrave, o qual se refere à fragilidade dentro da estrutura governamental, o que as torna mais vulneráveis a mudanças políticas e cortes orçamentários, sendo pouco prestigiadas na divisão dos recursos, o que significa fluxo de verbas insuficiente e irregular (Salgado; Nogueira, 2001).

A situação não é grave apenas no Brasil. O déficit de financiamento é, se não o mais relevante, um dos maiores problemas enfrentados pelas áreas protegidas no mundo. A falha do setor ambiental é praticamente universal, independentemente do grau de desenvolvimento dos países, entretanto, a escassez de recursos é uma restrição mais intensa nos países em desenvolvimento (IUCN, 2016a).

A urgência em cuidar de agendas como a da diminuição da pobreza faz com que outros temas importantes sejam postergados. Logo, há evidências de recursos governamentais designados à conservação que são desviados para assuntos politicamente mais sensíveis nesses países (IUCN, 2016a). A questão ambiental acaba, na maioria das vezes, encarada de forma secundária e/ou excluída da pauta governamental se confrontada às necessidades de outros setores tão importantes, como saúde e educação.

A melhoria da sustentabilidade financeira das UC está relacionada à capacidade de garantir recursos financeiros suficientes, estáveis e de longo prazo, e reparti-los em tempo hábil e de forma adequada, para cobrir os custos totais das áreas protegidas e garantir que sejam geridas com eficácia e eficiência quanto à conservação e demais objetivos (Emerton; Bishop; Thomas, 2006).

Ressalta-se, portanto, que taxas, ingressos ou concessão de serviços arrecadados pelas UC são alocados no Tesouro Nacional, que muitas vezes dá outro destino aos recursos gerados pela UC, e agrava o tempo de repasse do mesmo para manter em boas condições a gestão da área natural. 
A atividade turística no Brasil vem sendo movimentada cada vez mais pelo segmento turismo de natureza, que depende da proteção de paisagens naturais, as quais cumprem funções cujos benefícios são usufruídos por grande parte da população brasileira, inclusive por setores econômicos em contínuo crescimento, os quais nem se dão conta dos benefícios (Medeiros et al., 2011).

O aumento no fluxo de turistas nas áreas protegidas em parte se deve a contabilização de áreas nas quais não havia anteriormente nenhum tipo de registro ou acompanhamento. O número de visitantes nos parques nacionais brasileiros cresceu em 337\% no período de 2006 a 2016 (ICMBio, 2016).

Cabe salientar que o crescimento do número de visitantes nos Parques Nacionais brasileiros representa um forte e importante impacto na economia local, gerando empregos, renda e valorização dos ativos ambientais. Nesse contexto, para a economia brasileira, a visitação tem potencial para gerar recursos significativos para as UC, uma vez que há um aumento do interesse dos turistas por atividades recreativas em ambientes naturais (Medeiros et al., 2011).

\section{A VALORAÇÃO ECONÔMICA AMBIENTAL}

A valoração econômica do meio ambiente é um instrumento chave na gestão ambiental, e consiste em atribuir um valor monetário ao meio ambiente, por meio da aplicação de métodos econômicos (Motta, 1997), uma vez que mede as preferências das pessoas por um recurso ou serviço ambiental, e assim estima os custos sociais de se usar esses recursos escassos, ou incorporar os benefícios sociais advindos do uso desses recursos (Veiga; Ehlers, 2003).

Nesse sentido, os valores atribuídos aos ecossistemas e à biodiversidade são compostos pelos valores de uso e valores de não uso, os quais somados formam o Valor Econômico Total - VET (Guedes; Seehusen, 2011; Motta, 2006).

Os valores de uso são atribuídos pela sociedade a um recurso natural pelo seu uso efetivo no presente ou uso potencial no futuro, e é constituído de valores de uso direto, indireto e de opção (Parker et al., 2012). Primeiramente, o valor de uso direto relaciona-se ao uso direto do recurso natural. Considerando que um mesmo recurso natural possa ter vários usos distintos, este poderá ter vários valores de uso direto (Guedes; Seehusen, 2011; Motta, 2006; Parker et al., 2012; Toledo, 1998).

Por exemplo, o uso direto de uma floresta pode ser a extração de madeira ou o consumo de frutos, e em um parque nacional a visitação. Logo, os serviços de beleza cênica para atividades turísticas ou recreacionais em UC têm um valor de uso direto.

Além do valor de uso direto, o valor de uso indireto dos recursos naturais relaciona-se às funções dos ecossistemas que beneficiam as pessoas indiretamente. Como exemplos, purificação da 
água, contenção da erosão, formação do solo, ciclagem de nutrientes, polinização, controle biológico e regulação da composição dos gases atmosféricos (Guedes; Seehusen, 2011; Motta, 2006; Toledo, 1998).

E o valor de opção representa o ato de deixar uma opção para ser usada futuramente. Por exemplo, manter áreas naturais para no futuro obter os benefícios advindos de produtos originados de recursos genéticos ainda não descobertos de plantas e animais tropicais (Guedes; Seehusen, 2011; Motta, 2006; Parker et al., 2012; Toledo, 1998).

Os valores de não uso são separados em valores de existência e de legado (Parker et al., 2012). O valor de existência não deve estar relacionado a qualquer uso efetivo no presente ou uso potencial no futuro do recurso natural. Relaciona-se, assim, ao conceito de valor intrínseco dos recursos naturais. Esse valor expressa os elementos éticos e morais da sociedade, representado pela disposição a pagar das pessoas para a conservação de recursos naturais e a biodiversidade, independentemente de que sejam utilizados ou não (Pearce; Turner, 1990; Toledo, 1998).

A conservação dos recursos naturais pode ser um bem por si mesmo para a sociedade. Isso se relaciona ao valor de existência, e também pode significar uma herança para as futuras gerações, que é traduzido como valor de herança ou de legado (Parker et al., 2012; Pearce; Turner, 1990; Toledo, 1998).

Neste sentido, tem sido proposto um conjunto de elementos para justificar o valor de existência, sintetizado em alguma forma de altruísmo do ser humano, no sentido de cuidar de outros seres vivos (Motta, 2006; Parker et al., 2012; Pearce; Turner, 1990). Como exemplo tem-se a disposição a pagar para que uma espécie seja protegida em seu habitat natural, mesmo que o pagador saiba que nunca verá a espécie (Guedes; Seehusen, 2011).

E o valor de legado se refere ao valor atribuído a algo para que ele seja conservado, permitindo que próximas gerações dele se beneficiem pelo uso ou não uso (Guedes; Seehusen, 2011; Parker et al., 2012).

A biodiversidade possui um valor intrínseco atribuído pelas pessoas, assim como valores de opção. Apesar de muitas pessoas se beneficiarem das ações e atividades que levam à perda de biodiversidade e mudanças nos ecossistemas, os custos sofridos pela sociedade por tais mudanças é muitas vezes maior (Millennium Ecossystem Assessment, 2005).

Nesse sentido, a movimentação econômica nas regiões favorecidas por suas belezas cênicas pode gerar benefícios adicionais de distribuição de renda para as populações locais e do entorno, estando muitas vezes as UC localizadas em áreas menos desenvolvidas (Guedes; Seehusen, 2011).

Estudos têm buscado valorar os benefícios econômicos providos pelos ecossistemas e seus serviços ambientais para a sociedade, dando mais clareza sobre os ganhos e as perdas que cada 
alternativa envolve. Nesse contexto, os valores econômicos dos serviços ambientais permitem mensurar e comparar a outros bens produzidos ou recursos financeiros (TEEB, 2010). No caso da visitação em parques nacionais, mede-se o valor econômico de uso direto voltado para a recreação; e os valores de não uso, que são os de existência e de legado.

Dentre os métodos de valoração ambiental existentes, dois deles são amplamente aplicados em casos de recreação em parques nacionais (Sinclair; Stabler, 2009), o Método de Custo de Viagem - MCV e o Método de Valoração Contingente - MVC.

O custo de viagem é o valor total das despesas que cada indivíduo gasta para visitar o parque, isto é, deve considerar, por exemplo, os gastos relativos com transporte para se chegar ao parque e regressar à cidade de origem, hospedagem, alimentação, compras de mercado, taxas de ingresso e compra de souvenirs (Maia; Romeiro, 2008; Serra et al., 2004; Sinclair; Stabler, 2009)

Foram investigados diversos trabalhos de valoração ambiental em áreas naturais, no Brasil e em outros países, assim, no Quadro 1 são apresentadas variáveis estatisticamente significativas de alguns estudos que aplicaram o Método de Custo de Viagem. Cabe salientar que os sinais positivos (+) mostram uma relação direta com os gastos efetivos da viagem, enquanto que os sinais negativos (-) indicam que há uma relação inversa em relação aos gastos efetivos da viagem.

O MVC consiste em determinar o valor da disposição a pagar declarada pelos entrevistados, em que estes revelam as suas preferências, permitindo, assim, a construção de um mercado hipotético para o recurso natural (Mota, 2004; Sinclair; Stabler, 2009). Além disso, pretende-se identificar as variáveis mais relevantes na determinação da disposição a pagar dos visitantes do PNCV pelos serviços ambientais. Esse método possibilita que valores econômicos sejam estimados para captar os valores de opção e de existência (Ekins, 1993).

Foram investigados diversos trabalhos de valoração ambiental no Brasil e em outros países os quais aplicaram o MVC. As variáveis estatisticamente significativas de alguns estudos realizados sobre o assunto estão apresentadas no Quadro 2.

\section{CONSIDERAÇÕES FINAIS}

Vale lembrar que uma condição primordial para a obtenção de recursos financeiros públicos para a preservação das áreas protegidas é a capacidade dos planejadores e gestores para justificar as necessidades e objetivos socioeconômicos de cada localidade. De tal modo, estudos sobre a valoração econômica ambiental são necessários para convencer os governos nacionais e estrangeiros a cumprirem os compromissos de financiamento das áreas protegidas.

Além disso, observou-se em pesquisas realizadas no Brasil e no exterior a relevância do tema ambiental para fortalecer os argumentos com vistas à proteção dos parques nacionais, assunto esse 
que vem causando preocupação aos formuladores de políticas, planejadores e pesquisadores. E também subsidiar e incentivar políticas públicas que estimulem formas mais sustentáveis de uso do solo, utilizando-se de ferramentas econômicas para a valoração das UC, especialmente no Brasil.

Dessa forma, dentre os desafios ambientais estão o desenvolvimento de mecanismos inovadores de financiamento das áreas, e assim incrementar os recursos financeiros e também estimular melhorias na gestão e sustentabilidade das áreas protegidas.

A tendência de aumento na procura por atividades recreativas em ambientes naturais e a estruturação dos Parques Nacionais, há potencial de incremento dos benefícios decorrentes do turismo, tanto para as economias locais quanto para atender as necessidades financeiras de manutenção dessas áreas.

\section{REFERÊNCIAS}

ADAMS, C. et al. Valoração econômica do Parque Estadual Morro do Diabo (SP). São Paulo: Páginas \& Letras, 2003.

ALVAREZ S., LARKIN S. L. Valuing ecological restoration and recreational benefits in a mountain protected area: The case of Los Nevados National Park, Colombia. Journal of Sustainable Development, v. 3, n. 4, p. 3-16, dez. 2010.

ARAÚJO, M. A. R. Unidades de conservação: importância e história no mundo. In: NEXUCS (Org.). Unidades de Conservação no Brasil: o caminho da gestão para resultados. São Paulo: RiMa, 2012.

ARAÚJO, I. T. de. Disposição a pagar pela recuperação/preservação da caatinga no município de Mossoró-RN. Dissertação (Mestrado em Ambiente, Tecnologia e Sociedade) - Programa de pós-graduação em ambiente, tecnologia e sociedade, Universidade Federal Rural do Semi-Árido, Mossoró, 2013.

BARAL, N.; STERN, M. J.; BHATTARAI, R. Contingent valuation of ecotourism in Annapurna conservation area, Nepal: Implications for sustainable park finance and local development. Ecological Economics, v. 66, n. 2-3, p. 218-227, jun. 2008.

BRASIL. Constituição da República Federativa do Brasil, promulgada em 05 de outubro de 1988.

Lei n. 617, de 18 de agosto de 2000. Dispõe sobre o Plano Diretor Urbano, Rural e Ambiental do Munícipio de de Alto Paraíso de Goiás, Estado de Goiás. 2000a.

. Lei n. 9.985, de 18 de julho de 2000. Regulamenta o art. 225, § 1o, incisos I, II, III e VII da Constituição Federal, institui o Sistema Nacional de Unidades de Conservação da Natureza e dá outras providências. $2000 \mathrm{~b}$.

. Lei n. 11.516, de 28 de agosto de 2007. Dispõe sobre a criação do Instituto Chico Mendes de Conservação da Biodiversidade e dá outras providências. 2007.

Pilares para a Sustentabilidade Financeira do Sistema Nacional de Unidades de Conservação. Brasília: MMA, 2009.

CAMPHORA, A. L. Sustentabilidade econômica das áreas protegidas no brasil: lacunas institucionais para a consolidação de alternativas. Revista Opera, v. 9, p. 155-170, abr. 2010. 
DOMICIANO, C. S. Valores ambientais e desenvolvimento: um estudo de caso do distrito de São Jorge e do Parque Nacional da Chapada dos Veadeiros, 2014. Universidade Federal de Goiás.Tese (Doutorado em Ciências Ambientais). Programa de Pós-Graduação em Ciências Ambientais, Universidade Federal de Goiás, 2014.

DOWNWARD, P.; LUMSDON, L. Tourism Transport and Visitor Spending: a study in the North York Moors National Park, UK. Journal of Travel Research, v. 42, n. 4, p. 415-420, 2004.

DRIML, S. The Economic Value of Tourism to National Parks and Protected Areas in Australia. CRC for Sustainable Tourism, 2010.

EAGLES, P. F.; HILLEL, O. Improving protected area finance through tourism. In: SECRETARIAT OF THE CONVENTION ON BIOLOGICAL DIVERSITY. Protected Areas in Today's World: Their Values and Benefits for the Welfare of the Planet. 36. ed. Montreal: CDB, 2008.

EHRENFELD, D. Por que atribuir um valor à biodiversidade? In: WILSON, E. O. (Org.). Biodiversidade. Rio de Janeiro: Nova Fronteira, 1997.

EKINS, P. Economic Values and the Natural World. David Pearce, Review. International Affairs, v. 69, n. 4, p. 774-775, 1993.

EMERTON, L.; BISHOP, J.; THOMAS, L. Sustainable financing of protected areas.: A global review of challenges and options. Best Practice Protected Area Guidelines Series n. 13, IUCN, 2006.

EMIRIYA, M.; ROBSON, M.; GOMBARAGO, E. N. T. An application of the individual travel cost method to Nyanga National Park, Zimbabwe. Researchjournali's Journal of Economics, v. 1, n. 1, p. 1-16, nov. 2013.

EZEBILO, E. Willingness to Pay for Maintenance of a Nature Conservation Area: A Case of Mount Wilhelm, Papua New Guinea. Asian Social Science, v. 12, n. 9, p. 149-161, 2016.

FONSECA, M.; LAMAS, I.; KASECKER, T. O Papel das Unidades de Conservação. In: Brasil, maior biodiversidade do mundo. Scientific American Brasil, n.39, p. 18-23, maio 2010.

GUEDES, F. B.; SEEHUSEN, S. E. (Org.). Pagamentos por Serviços Ambientais na Mata Atlântica: lições aprendidas e desafios. Brasília: MMA, 2011.

HADKER, N.; et al. Willingness to pay for Borivli National Park: evidence from a contingent valuation. Ecological Economics, v. 21, n. 2, p. 105-122, 1997.

HILDEBRAND, E. Avaliação econômica dos benefícios gerados pelos parques urbanos: estudo de caso em Curitiba-PR Tese (Doutorado em Ciências Florestais). Programa de Pós-Graduação em Engenharia Florestal, Universidade Federal do Paraná, Curitiba, 2001.

HOA, D. Le; LY, N. T. Y. Willingness to Pay for the Preservation of Lo Go-Xa Mat National Park in Vietnam. Economy and Environmental Program for Southeast Asia, Nov. 2009.

ICMBIO, 2016. Ranking de visitantes Parques Nacionais 2012-2014. Disponível em: $<$ http://www.icmbio.gov.br/portal/images/stories/o-quefazemos/ranking visitantes PN 2012 a 2014.pdf>. Acesso em: 14 jan. 2016.

IUCN, 2016a. Disponível em: <http://www.iucn.org/>. Acesso em: 4 fev. 2016. fev. 2016.

Protected Planet, 2016b. Disponível em: <http://blog.protectedplanet.net/>. Acesso em: 10 
KASTENHOLZ, E. Analysing determinants of visitor spending for the rural tourist market in North Portugal. Tourism Economics, v. 11, n. 4, p. 555-569, 2005.

KRUGER, M.; SAAYMAN, M.; ELLIS, S. M. Determinants of visitor expenditure at the Aardklop National Arts Festival. Event Management, v. 14, n. 2, p. 137-148, 2010.

KRUGER, M.; SAAYMAN, MANNERS, B. Determinants of visitor expenditure at the Tsitsikamma National Park. Journal of Economic and Financial Sciences, v. 5, n. 1, p. 11-30, Apr. 2012.

LEONES, J.; COLBY, B.; CRANDALL., K. Tracking expenditures of the elusive nature tourists of Southeastern Arizona. Journal of Travel Research, v. 36, n. 3, p. 56-64, 1998.

LONG, P. T.; PERDUE, R. R. The Economic Impact of Rural Festivals and Special Events: Assessing The Spatial Distribution of Expenditures. Journal of Travel Research, v. 28, n. 4, p. 10-14, 1990.

MAIA, A. G.; ROMEIRO, A. R. Validade e confiabilidade do Método de Custo de Viagem: um estudo aplicado ao Parque Nacional da Serra Geral. Economia Aplicada, São Paulo, v. 12, n. 1, p. 103-123, jan.-mar. 2008.

MARTINS, E. C. O turismo como alternativa de desenvolvimento sustentável: o caso de Jericoacoara no Ceará. Tese (Doutorado em Ciências). Escola Superior de Agricultura Luiz de Queiroz, Universidade de São Paulo, Piracicaba, 2002.

MEDEIROS, R. et al. (Ed.). Contribuição das unidades de conservação para a economia nacional: Sumário Executivo. Brasília: UNEP/WCMC, 2011.

; IRVING, M.; GARAY, I. A Proteção da Natureza no Brasil: evolução e conflitos de um modelo em construção. Revista de Desenvolvimento Econômico, ano VI, n. 9, p. 83-93, 2004.

MEHMETOGLU, M. Typologising nature-based tourists by activity: Theoretical and practical implications. Tourism Management, v. 28, n. 3, p. 651-660, 2007.

MILLENNIUM ECOSYSTEM ASSESSMENT. Ecosystems and human well-being: biodiversity synthesis. Washington: World Resources Institute, 2005.

MOTA, J. A. Economia, meio ambiente e sustentabilidade: as limitações do mercado onde o mercado é o limite. Boletim Científico ESMPU, v. 3, n. 12, p. 67-87, jul.-set. 2004.

MOTTA, R. S. da. Manual para valoração econômica de recursos naturais. Rio de Janeiro: IPEA/MMA/PNUD/CNPq, 1997.

Economia Ambiental. Rio de Janeiro: FGV, 2006.

MUÑOZ, J. P. M. Valoração Econômica do Parque Nacional de Brasília. Dissertação (Mestrado em Manejo Florestal). Programa de Pós-Graduação em Ciências Florestais, Universidade de Brasília, Brasília, 2015.

PARDINI, H. Estudo de caso 5.1: O desafio do uso público nas Unidades de Conservação Brasileiras. In: NEXUCS (Org.). Unidades de Conservação no Brasil: o caminho da gestão para resultados. RiMa, 2012.

PARKER, C.; CRANFORD, M.; OAKES, N.; LEGGETT, M. The Little Biodiversity Finance Book: a guide to proactive investment in natural capital (PINC). Oxford: Global Canopy Programme, 2012.

PEARCE, D. W.; TURNER, R. K. Economics of natural resources and the environment. Baltimore: The Johns Hopkins Univesity Press, 1990.

PRIMACK, R. B.; RODRIGUES, E. Biologia da conservação. Londrina: Planta, 2001. 
RYLANDS, A. B.; BRANDON, K. Brazilian protected areas. Conservation Biology, v. 19, n. 3, p. 612-618, jun. 2005.

RODRIGUES, C. G. O; GODOY, L. R. C. Atuação pública e privada na gestão de Unidades de Conservação : aspectos socioeconômicos da prestação de serviços de apoio à visitação em parques nacionais. Public and private policy in the management of protected areas: socioeconomic aspects in the rendering of support services to visitors in national parks. Desenvolvimento e Meio Ambiente, $\mathrm{v}$. 28, p. 75-88, 2013.

SAAYMAN, A.; SAAYMAN, M. Determinants of spending at two South African Marine National Parks. Journal of Tourism Research \& Hospitality, v. 3, n. 2, 2014.

SAAYMAN, M.; SAAYMAN, A. Determinants of spending: an evaluation of three major sporting events. International Journal of Tourism Research, v. 14, p. 124-138, 2012.

SALGADO, G. S. M.; NOGUEIRA, J. M. Economia e gestão de áreas protegidas: o caso do Parque Nacional de Brasília. In: IV Encontro da Sociedade Brasileira de Economia Ecológica. Belém: PIEBTUFPA-. FADESP, 2001.

SANTANA, R. F.; MOTA, J. A. O valor econômico de existência do Parque Nacional do Jaú (Amazonas). Revista de Economia, Paraná, v. 30, n. 1, p. 49-63, 2004.

SERRA, M. A et al. A valoração contingente como ferramenta de economia aplicada à conservação ambiental: o caso da Estrada Parque Pantanal. Planejamento e políticas públicas, n. 27, p. 193-212, 2004.

SHRESTHA, R. K.; SEIDL, A. F.; MORAES, A. S. Value of recreational fishing in the Brazilian Pantanal : a travel cost analysis using count data models. Ecological Economics, v. 42, n. 1, p. 289-299, 2002.

SIEW, M. K. et al. Estimating Willingness to Pay for Wetland Conservation: A Contingent Valuation Study of Paya Indah Wetland, Selangor Malaysia. Procedia Environmental Sciences, v. 30, p. 268-272, 2015.

SINCLAIR, M. T.; STABLER, M. Aspectos econômicos do turismo. Brasília: Universidade de Brasília, 2009.

SILVA, Rubicleis Gomes da; LIMA, João Eustáquio de. Valoração contingente do Parque "Chico Mendes": uma aplicação probabilística do método Referendum com bidding games. Revista de Economia e Sociologia Rural, Rio de Janeiro, vol. 42, n. 04, p. 685-708, out/dez 2004.

SOUSA, G. B. DE; MOTA, J. A. Valoração econômica de áreas de recreação o caso do Parque Metropolitano de Pituaçu, Salvador, BA. Revista de Economia, v. 32, n. 1, p. 37-55, 2006.

SPOTTS, D. M.; MAHONEY, E. M. Segmenting visitors to a destination region based on the volume of their expenditures. Journal of Travel Research, v. 29, n. 4, p. 24-31, 1991.

TAYLOR, D. T.; FLETCHER, R. R.; CLABAUGH, T. A comparison of characteristics, regional expenditures, and economic impact of visitors to historical sites with other recreational visitors. Journal of Travel Research, v. 32, n. 1, p. 30-35, 1993.

TEEB. The Economics of Ecosystems and Biodiversity: The Ecological and Economic Foundations. UNEP/Earthprint, 2010.

THRANE, C. Jazz Festival visitors and their expenditures: linking spending patterns to musical interest. Journal of Travel Research, v. 40, n. 3, p. 281-286, 2002.

TOLEDO, Alejandro. Economía de la biodiversidad. Red de Formación Ambiental, 1998.

VEIGA, J. E.; EHLERS, E. Diversidade biológica e dinamismo econômico no meio rural. In: MAY, P. H.; LUSTOSA, M. C.; VINHA, V. (Org.). Economia do meio ambiente: teoria e prática. Rio de Janeiro: Elsevier, p. 271-289, 2003.

WEARING, S.; NEIL, J. Ecoturismo: impactos, potencialidades e possibilidades. Barueri, SP: Manole, 2014. 
Quadro 1: Estudos sobre o Método de Custo de Viagem e variáveis preditoras significantes que explicam os gastos efetivos dos visitantes.

\begin{tabular}{|c|c|c|}
\hline RECURSO VALORADO & VARIÁVEIS PREDITORAS & REFERÊNCIAS \\
\hline Pantanal, Brasil & $\begin{array}{l}\text { Espécies de peixes (+), proximidade do local } \\
(+) \text {, viagem motivada pelo valor de não uso } \\
(+)\end{array}$ & $\begin{array}{l}\text { (SHRESTHA; SEIDL; } \\
\text { MORAES, 2002) }\end{array}$ \\
\hline $\begin{array}{l}\text { Parque Nacional da Serra Geral } \\
\text { Santa Catarina e Rio Grande do Sul }\end{array}$ & escolaridade (+) & (MAIA; ROMEIRO, 2008) \\
\hline $\begin{array}{l}\text { Parque Nacional da Chapada dos } \\
\text { Veadeiros }\end{array}$ & $\begin{array}{l}\text { idade }(+) \text {, renda }(+) \text {, grupo }(+) \text {, proximidade } \\
(-) \text {, escolaridade }(+)\end{array}$ & (DOMICINIANO, 2014) \\
\hline $\begin{array}{l}\text { Parques e Bosques Urbanos } \\
\text { Curitiba }\end{array}$ & renda $(+)$ & HILDEBRAND, 2001 \\
\hline $\begin{array}{l}\text { Parque Nacional Tsitsikamma e Parque } \\
\text { Nacional Wilderness, África do Sul }\end{array}$ & $\begin{array}{l}\text { renda }(-) \text {, idade }(+) \text {, estado civil casado }(-) \text {, } \\
\text { tempo de permanência } \\
(-) \text {, motivos da visita ao Parque }(+) \text {, viagem } \\
\text { com crianças }(-) \text {, } \\
\text { preferência por determinadas espécies } \\
\text { marinhas }(+)\end{array}$ & $\begin{array}{l}\text { (SAAYMAN; SAAYMAN, } \\
\text { 2014) }\end{array}$ \\
\hline $\begin{array}{l}\text { Parque Nacional Tsitsikamma, África do } \\
\text { Sul }\end{array}$ & $\begin{array}{l}\text { grupo de viagem (-), tempo de permanência } \\
(+) \text {, informações do Parque em revistas (+) }\end{array}$ & $\begin{array}{l}\text { (KRUGER; SAAYMAN; } \\
\text { MANNERS, 2012) }\end{array}$ \\
\hline $\begin{array}{l}\text { Parque Nacional Nyanga (NNP), } \\
\text { Zimbábue }\end{array}$ & $\begin{array}{l}\text { número de visitas (-), gasto com } \\
\text { deslocamento (-), renda (-), lugares } \\
\text { substitutos (-) }\end{array}$ & $\begin{array}{l}\text { (EMIRIYA; ROBSON; } \\
\text { GOMBARAGO, 2013) }\end{array}$ \\
\hline $\begin{array}{l}\text { Parque Nacional Los Nevados, } \\
\text { Colômbia }\end{array}$ & número de visitas (-) & (ALVAREZ; LARKIN, 2010) \\
\hline $\begin{array}{l}\text { Região de Turismo de natureza e } \\
\text { recreação ao ar livre } \\
\text { Pictured Rocks National Lakeshore e } \\
\text { Tahquamenon Falls State Park são os } \\
\text { principais atrativos } \\
\text { Estados Unidos }\end{array}$ & $\begin{array}{l}\text { Viagem com filhos }(+) \text {, tempo de } \\
\text { permanência }(+) \text {, visita mais atrativos }(+) \text {, } \\
\text { mais atividades de lazer }(+)\end{array}$ & (SPOTTS; MAHONEY, 1991) \\
\hline $\begin{array}{l}\text { Turismo de natureza e de locais } \\
\text { históricos } \\
\text { Bighorn National Forest é o principal } \\
\text { atrativo } \\
\text { Estados Unidos }\end{array}$ & $\begin{array}{l}\text { renda familiar }(+) \text {, } \\
\text { tamanho do grupo }(+) \text {, tempo de } \\
\text { permanência }(-) \text {, hospedagem em } \\
\text { acampamento }(-)\end{array}$ & $\begin{array}{l}\text { (TAYLOR; FLETCHER; } \\
\text { CLABAUGH, 1993) }\end{array}$ \\
\hline $\begin{array}{l}\text { Turismo de natureza no Ramsey Canyon } \\
\text { Preserve e na Área de Conservação } \\
\text { Nacional de San Pedro Riparian } \\
\text { Arizona, Estados Unidos }\end{array}$ & $\begin{array}{l}\text { visita mais atrativos }(+) \text {, } \\
\text { tempo de permanência }(-) \text {, } \\
\text { proximidade }(+)\end{array}$ & $\begin{array}{l}\text { (LEONES; COLBY; } \\
\text { CRANDALL., 1998) }\end{array}$ \\
\hline $\begin{array}{l}\text { North York Moors } \\
\text { National Park } \\
\text { Reino Unido }\end{array}$ & $\begin{array}{l}\text { viagem de carro }(+) \text {, } \\
\text { viagem de transporte público }(+) \text {, tamanho } \\
\text { do grupo }(+) \text {, } \\
\text { tempo de permanência }(+)\end{array}$ & $\begin{array}{l}\text { (DOWNWARD; LUMSDON, } \\
\text { 2004) }\end{array}$ \\
\hline $\begin{array}{l}\text { Turismo de natureza } \\
\text { Finlândia }\end{array}$ & $\begin{array}{l}\text { idade }(-) \text {,tempo de permanência }(+) \text {, } \\
\text { distância }(+) \text {, } \\
\text { frequência de visitas }(-) \\
\text { áreas privadas }(+) \text {, } \\
\text { atividades de esqui }(+) \text {, infraestrutura para } \\
\text { camping }(+) \text {, colheita de alimentos }(-) \text {, } \\
\text { hospedagem em casa de família }(-)\end{array}$ & $\begin{array}{l}\text { (POUTA; NEUVONEN; } \\
\text { SIEVÄNEN, 2006) }\end{array}$ \\
\hline $\begin{array}{l}\text { Parque Nacional Table Mountain } \\
\text { (TMNP) } \\
\text { África do Sul }\end{array}$ & idade (-) & (CINI; SAAYMAN, 2014) \\
\hline
\end{tabular}

(+) há relação direta com os gastos efetivos da viagem

(-) há relação inversa com os gastos efetivos da viagem

Fonte: Elaborado pela autora. 
Quadro 2: Estudos sobre o Método de Valoração Contigente e variáveis preditoras significantes que explicam a disposição a pagar dos visitantes/população.

\begin{tabular}{|c|c|c|c|}
\hline $\begin{array}{l}\text { RECURSO } \\
\text { VALORADO }\end{array}$ & $\begin{array}{c}\text { VALOR } \\
\text { ECONÔMICO }\end{array}$ & VARIÁVEIS PREDITORAS & REFERÊNCIAS \\
\hline $\begin{array}{l}\text { Parque Nacional do } \\
\text { Jaú, Amazonas } \\
\text { Brasil }\end{array}$ & Valor de não uso & $\begin{array}{l}\text { renda familiar }(+) \text {, idade }(+) \text {, } \\
\text { nível de importância da existência do PNJ (+) }\end{array}$ & $\begin{array}{l}\text { (SANTANA; MOTA, } \\
\text { 2004) }\end{array}$ \\
\hline $\begin{array}{l}\text { Parque } \\
\text { Metropolitano de } \\
\text { Pituaçu } \\
\text { Salvador, Bahia }\end{array}$ & Valor de não uso & $\begin{array}{l}\text { Moram mais próximo ao Parque }(+) \text {, } \\
\text { escolaridade }(+) \text {, } \\
\text { anos de frequência ao local }(+) \text {, número de } \\
\text { visitas mensais }(+) \text {, preocupação com os } \\
\text { problemas ambientais }(+)\end{array}$ & (SOUSA; MOTA, 2006) \\
\hline $\begin{array}{l}\text { Caatinga } \\
\text { Mossoró-RN }\end{array}$ & Valor de não uso & $\begin{array}{l}\text { renda }(+) \text {, reciclagem }(-) \text {, } \\
\text { escolaridade }(-) \text {, dano ambiental }(-)\end{array}$ & (ARAÚJO, 2013) \\
\hline $\begin{array}{l}\text { Parque Nacional de } \\
\text { Brasília }\end{array}$ & Valor de não uso & $\begin{array}{l}\text { Renda }(+) \text {, conhecimento sobre o parque e meio } \\
\text { ambiente }(+) \text {, idade }(+)\end{array}$ & (MUÑOZ, 2015) \\
\hline $\begin{array}{l}\text { Parque Estadual } \\
\text { Morro do Diabo } \\
\text { São Paulo }\end{array}$ & Valor de não uso & $\begin{array}{l}\text { Renda }(+) \text {, idade }-16 \text { a } 24 \text { anos }(+) \text {, escolaridade } \\
-2 \text { o grau }(+)\end{array}$ & (ADAMS et al., 2003) \\
\hline $\begin{array}{l}\text { Parque Nacional da } \\
\text { Chapada dos } \\
\text { Veadeiros }\end{array}$ & Valor de uso & idade $(+)$, escolaridade $(+)$ & (DOMICINIANO, 2014) \\
\hline $\begin{array}{l}\text { Parque Chico } \\
\text { Mendes } \\
\text { Rio Branco, Acre } \\
\end{array}$ & Valor de não uso & renda $(+)$, escolaridade $(+)$, idade $(+)$ & (SILVA; LIMA, 2004) \\
\hline $\begin{array}{l}\text { Parque Nacional de } \\
\text { Brasília }\end{array}$ & Valor de não uso & $\begin{array}{l}\text { renda }(+) \text {, conhecimento do bem ambiental }(+) \text {, } \\
\text { idade }(+)\end{array}$ & (MUÑOZ, 2015) \\
\hline $\begin{array}{l}\text { Praia de } \\
\text { Jericoacoara } \\
\text { Ceará }\end{array}$ & Valor de uso & $\begin{array}{l}\text { número de dias no local }(-) \text {, proximidade, } \\
\text { escolaridade }(+) \text {, número de visitas ao local (+) }\end{array}$ & MARTINS, 2002 \\
\hline $\begin{array}{l}\text { Parques e Bosques } \\
\text { Urbanos } \\
\text { Curitiba }\end{array}$ & Valor de uso & renda (-) & HILDEBRAND, 2001 \\
\hline $\begin{array}{l}\text { Área de } \\
\text { Conservação de } \\
\text { Annapurna } \\
\text { Nepal }\end{array}$ & $\begin{array}{l}\text { Valor de uso e de } \\
\text { não uso }\end{array}$ & $\begin{array}{l}\text { valor do lance }(-), \\
\text { tamanho da família }(-), \\
\text { satisfação geral do visitante }(+), \\
\text { uso de guia }(+) \text {, tamanho do grupo (+) }\end{array}$ & $\begin{array}{l}\text { (BARAL; STERN; } \\
\text { BHATTARAI, 2008) }\end{array}$ \\
\hline $\begin{array}{l}\text { Parque Nacional Los } \\
\text { Nevados } \\
\text { Colômbia }\end{array}$ & Valor de não uso & $\begin{array}{l}\text { entrevistados que visitam parques nacionais ou } \\
\text { áreas naturais regularmente }(+) \text {, grupos maiores } \\
(+) \text {, viajando com crianças }(+) \text {, idade }(-) \text {, renda } \\
(+) \text {, membros de organizações ambientais ou } \\
\text { doadores para essas áreas (+) }\end{array}$ & $\begin{array}{l}\text { (ALVAREZ; LARKIN, } \\
\text { 2010) }\end{array}$ \\
\hline $\begin{array}{l}\text { Parque Nacional } \\
\text { Borivli } \\
\text { Índia }\end{array}$ & Valor de não uso & $\begin{array}{l}\text { nível de escolaridade }(+) \text {, renda }(+) \text {, atitudes } \\
\text { voltadas para o meio ambiente }(+)\end{array}$ & (HADKER et al., 1997) \\
\hline $\begin{array}{l}\text { Monte Wilhelm } \\
\text { Papua Nova Guiné }\end{array}$ & Valor de não uso & $\begin{array}{l}\text { renda }(+) \text {, escolaridade }(+) \text {, importância das } \\
\text { florestas }(+) \text {, disposição de desistir de terras } \\
\text { para conservação }(+)\end{array}$ & (EZEBILO, 2016) \\
\hline $\begin{array}{l}\text { Paya Indah Wetland, } \\
\text { Selangor } \\
\text { Malásia }\end{array}$ & Valor de uso & preço $(-)$, renda $(+)$, distância $(-)$, escolaridade $(-)$ & (SIEW et al., 2015) \\
\hline $\begin{array}{l}\text { Parque Nacional Lo } \\
\text { Go - Xa Mat } \\
\text { Vietnã }\end{array}$ & Valor de não uso & $\begin{array}{l}\text { renda }(-), \\
\text { valor mensal da conta de luz }(+) \text {, escolaridade } \\
(+)\end{array}$ & (HOA; LY, 2009) \\
\hline
\end{tabular}

(+) há relação direta com a disposição a pagar dos visitantes

(-) há relação inversa com a disposição a pagar dos visitantes

Fonte: Elaborado pela autora. 